\title{
A Novel Ultrasound-Based Lower Extremity Motion Tracking System
}

\author{
Kenan Niu, Victor Sluiter, Jasper Homminga, \\ André Sprengers, and Nico Verdonschot
}

\begin{abstract}
Tracking joint motion of the lower extremity is important for human motion analysis. In this study, we present a novel ultrasoundbased motion tracking system for measuring three-dimensional (3D) position and orientation of the femur and tibia in 3D space and quantifying tibiofemoral kinematics under dynamic conditions. As ultrasound is capable of detecting underlying bone surface noninvasively through multiple layers of soft tissues, an integration of multiple A-mode ultrasound transducers with a conventional motion tracking system provides a new approach to track the motion of bone segments during dynamic conditions. To demonstrate the technical and clinical feasibilities of this concept, an in vivo experiment was conducted. For this purpose the kinematics of healthy individuals were determined in treadmill walking conditions and stair descending tasks. The results clearly demonstrated the potential of tracking skeletal motion of the lower extremity and measuring
\end{abstract}

K. Niu $(\varangle) \cdot$ V. Sluiter $\cdot$ J. Homminga Laboratory of Biomechanical Engineering, MIRA Institute, University of Twente, Enschede, the Netherlands
A. Sprengers $\cdot$ N. Verdonschot
Orthopaedic Research Lab, Radboud University Medical Center, Nijmegen, the Netherlands

six-degrees-of-freedom (6-DOF) tibiofemoral kinematics and related kinematic alterations caused by a variety of gait parameters. It was concluded that this prototyping system has great potential to measure human kinematics in an ambulant, non-radiative, and noninvasive manner.

\section{Keywords}

Joint motion tracking - A-mode ultrasound · Knee $\cdot$ Kinematics $\cdot$ Lower extremity $\cdot$ Gait analysis

\subsection{Introduction}

Measuring skeletal motion occurring in the human joints is important to understand the functions of human joints [1], to assist the pathological diagnoses [2] and to monitor the actual threedimensional (3D) positions of bone segments during surgeries [3] (e.g., total hip arthroplasty [4] (THA), total knee arthroplasty [5, 6] (TKA)) and to assess the outcomes of treatments [7, 8]. Skeletal kinematic data may be used in motion analyses combined with biomechanical modeling, e.g., musculoskeletal models for inverse 
dynamics approaches $[9,10]$. Hence, a valid representation of actual skeletal motion and an accurate skeletal kinematics estimation is important in the fields of orthopedic research and human motion analysis [11]. However, the fact is that human skeletal structures are not exposed to the outside environment but are surrounded by the soft tissues (the muscles, the fat, the skin, etc.). Therefore, an effective measuring technique that could directly or indirectly detect the motion of the bone is necessary to monitor and trace the movements of bone segments underlying the skin surface $[12,13]$.

Currently, skin-mounted markers are widely used in human motion analysis to estimate the motions of bones by assuming no relative motions between the skin and bone [14]. However, this method is subject to soft tissue artifacts (STA) because the markers attached on the skin cannot represent the actual motions of underlying bone segments [15]. It has been reported that STA can cause measurement errors of markers up to $30 \mathrm{~mm}$ in the thigh [16]. The propagation of STA to knee joint kinematics has been reported to lead to average rotational errors of up to $4.4^{\circ}$ and $13.1^{\circ}$ and average translational errors of up to $13.0 \mathrm{~mm}$ and $16.1 \mathrm{~mm}$ for walking and cutting motions, respectively [17]. Although many researchers attempted to compensate for the STA by computer modeling [13, 18-24], no significant improvement has been found in previous studies [12].

With the development of medical imaging technologies, fluoroscopic systems have been utilized to capture high accurate joint kinematics in the prosthetic measurement for TKA patients [25-27]. However, high cost, cumbersome setup, and limited field of view (FOV) impede routine usage in the clinical setting. Recently, several groups have been working on the development of mobile fluoroscopy systems [28, 29]. Although using a robotic trolley or gantry carrying the fluoroscopic system following the movement of subject extends the FOV, the radiation exposure to the subject remains inevitable. Recently advanced four-dimensional (4D) MRI [30-32] and CT [33, 34] techniques have been reported to track the bone motion and to quantify the respective joint kinematics inside the scanners [30-32]. The disadvantages of this method are the limited FOV, limited sample rate, and the inability to measure kinematics during daily activities.

Besides abovementioned image modalities, ultrasound serves as a noninvasive and nonradiative imaging method to observe the soft tissues and internal organs in various clinical applications [35]. In addition, ultrasound is also capable of detecting bone surfaces through multiple layers of soft tissues [36]. Utilization of an ultrasound transducer combined with a surgical navigation system to accomplish the intraoperative registration of bone segments has been reported in computer-assisted orthopedic surgeries [37-39]. Due to its capability of detecting a bone surface under dynamic motions, the combination of multiple ultrasound transducers with conventional motion capture markers provides a new approach to estimate the 3D positions and orientations of bone segments and to quantify related joint kinematics. The bone detections (i.e., depths from the skin to bone) accompanied with corresponding spatial positions (3D coordinates of the ultrasound transducers) provide sufficient information to reconstruct the $3 \mathrm{D}$ bone motion per time frame without the effect of STA that exists in skin-mounted marker measurements. In vitro validation of this concept has been investigated for the knee joint in a previous study [40], which showed a relative high accuracy on the estimated tibiofemoral kinematics. The comparison with conventional skin-mounted markers measurement also has been conducted to assess the performance against widely used skin markers measurements. The ultrasound tracking system showed high accuracy in estimated 3D bone positions and quantified six-degrees-of-freedom (6-DOF) joint kinematics (maximum root-meansquare (RMS) error $3.44^{\circ}$ for rotations and 4.88 $\mathrm{mm}$ for translations). However, to evaluate the capability of tracking knee joint motions and quantifying 6-DOF tibiofemoral kinematics of a variety of daily activities for living subjects is a crucial step to explore its technical and clinical implementation. 
The aim of this study was to demonstrate and assess the in vivo capability of our proposed ultrasound tracking system when healthy subjects performed several daily activities, including treadmill walking at three different speeds and stair descent. We expected that kinematics alterations caused by different imposed gait parameters could also be identified by the ultrasound tracking system.

\subsection{Methods}

\subsubsection{Participants}

Five subjects (five males, age $37 \pm 10$ years, height $180 \pm 8 \mathrm{~cm}$, weight $75.4 \pm 14.1 \mathrm{~kg}$ ) participated in this study. Although only one subject had a meniscus operation four years ago, there was no influence and/or complaints on performing exercises reflecting daily activities as conducted during this experiment. The other subjects have no history of injury, treatment, or disorder affecting knee and hip functions. All subjects gave written informed consent. Prior to the experiment, each subject had an MRI scan using a Philips INGENIA 3T (BEST, the Netherlands) with a voxel size of $0.5 \mathrm{~mm} \times$ $0.5 \mathrm{~mm} \times 1 \mathrm{~mm}$ at the Radiology Department of Academisch Medisch Centrum (AMC, Amsterdam, Netherlands). After the MRI scan, the obtained MRI images were segmented manually to generate subject-specific geometrical surface models of the femur and tibia using Mimics 17.0 (Materialise N.V., Leuven, Belgium), which were exported in STL format. The femoral and tibial anatomical reference frames (ARF) were then defined based on obtained bone geometries using a method previously described [41]. Approval of this study (2017-3578) was obtained from the Ethical Committee at the Radboud University Medical Center (RUMC).

\subsubsection{Ultrasound Tracking System}

The ultrasound tracking system consisted of a conventional motion tracking system and an ultrasound signal acquisition system. In this study, we used Visualeyez VZ4000v system (PTI Phoenix Technologies Inc., Vancouver, Canada) equipped with two trackers to provide spatial positioning (see Figs. 11.1 and 11.2) with less than $0.5 \mathrm{~mm}$ RMS error [42]. The ultrasound signal and marker positioning information was collected and synchronized in the Diagnostic Sonar FI Toolbox (Diagnostic Sonar Ltd, Livingston, UK) with $2.3 \mathrm{GHz}$ CPU (Intel Core i7-3610QE) and 8GB RAM with a custom acquisition program written by LabVIEW (National Instruments, Austin, Texas, USA). Thirty A-mode ultrasound transducers $(7.5 \mathrm{MHz}$,

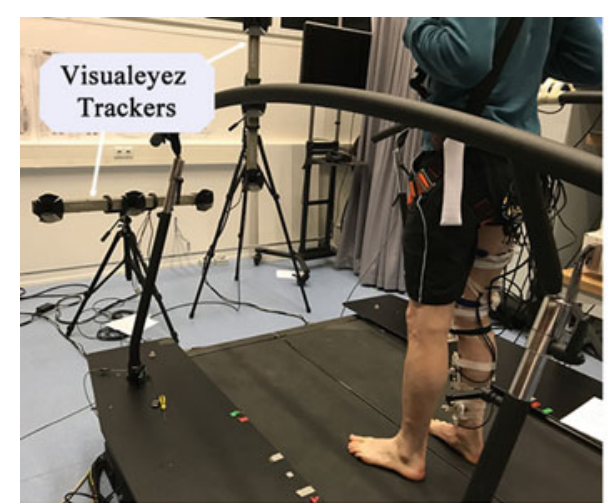

(a)

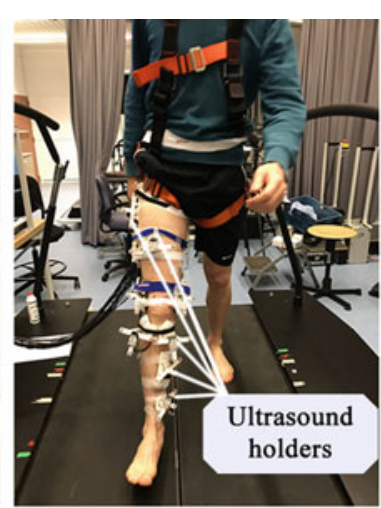

(b)

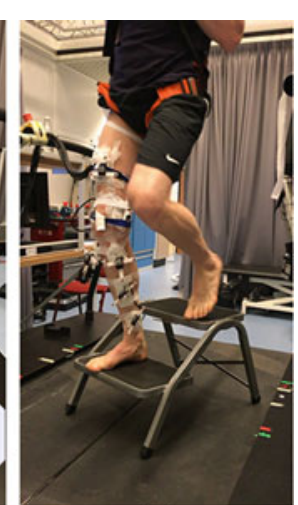

(c)
Fig. 11.1 (a) A side view of experimental setup, including two Visualeyez trackers to track the optical markers on ultrasound holder; (b) a front view of experimental setup, one subject wore all ultrasound holder and performed a treadmill walking task; (c) a subject performed stair descent with the ultrasound tracking system measurement 


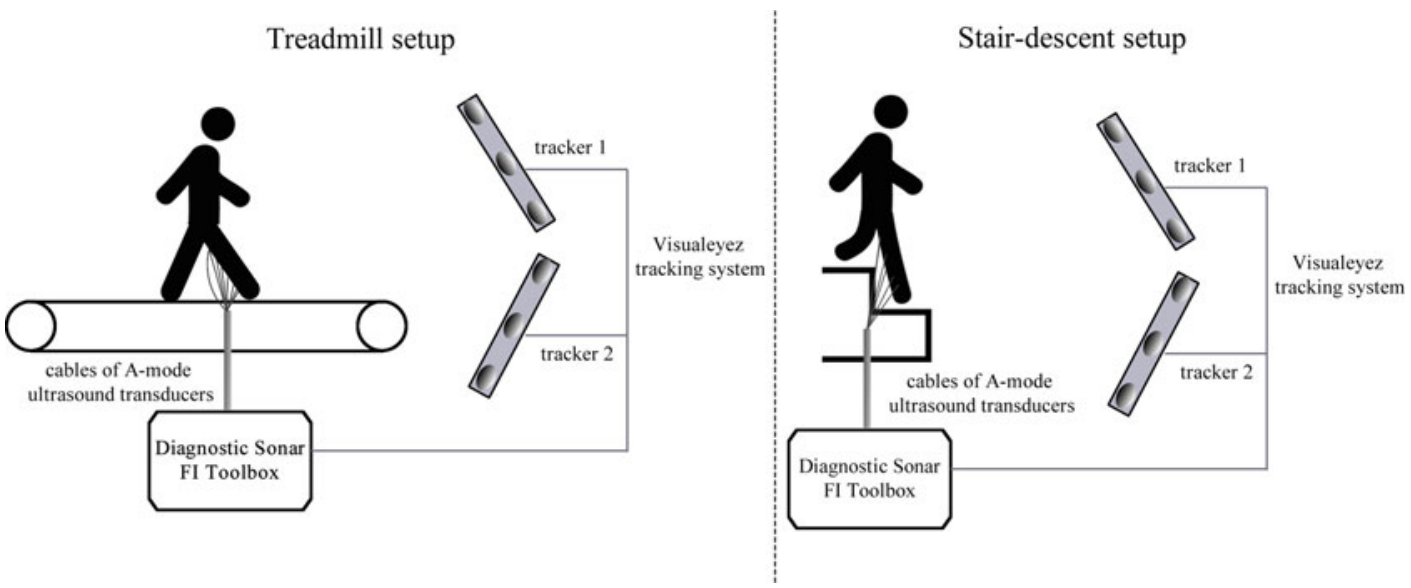

Fig. 11.2 A schematic representation of the experimental setups for treadmill walking (left) and stair descent (right). Two Visualeyez trackers were used to record the spatial information of attached ultrasound holders.
Diagnostic Sonar FI Toolbox received all raw ultrasound signals and was synchronized with collected spatial information from Visualeyez trackers

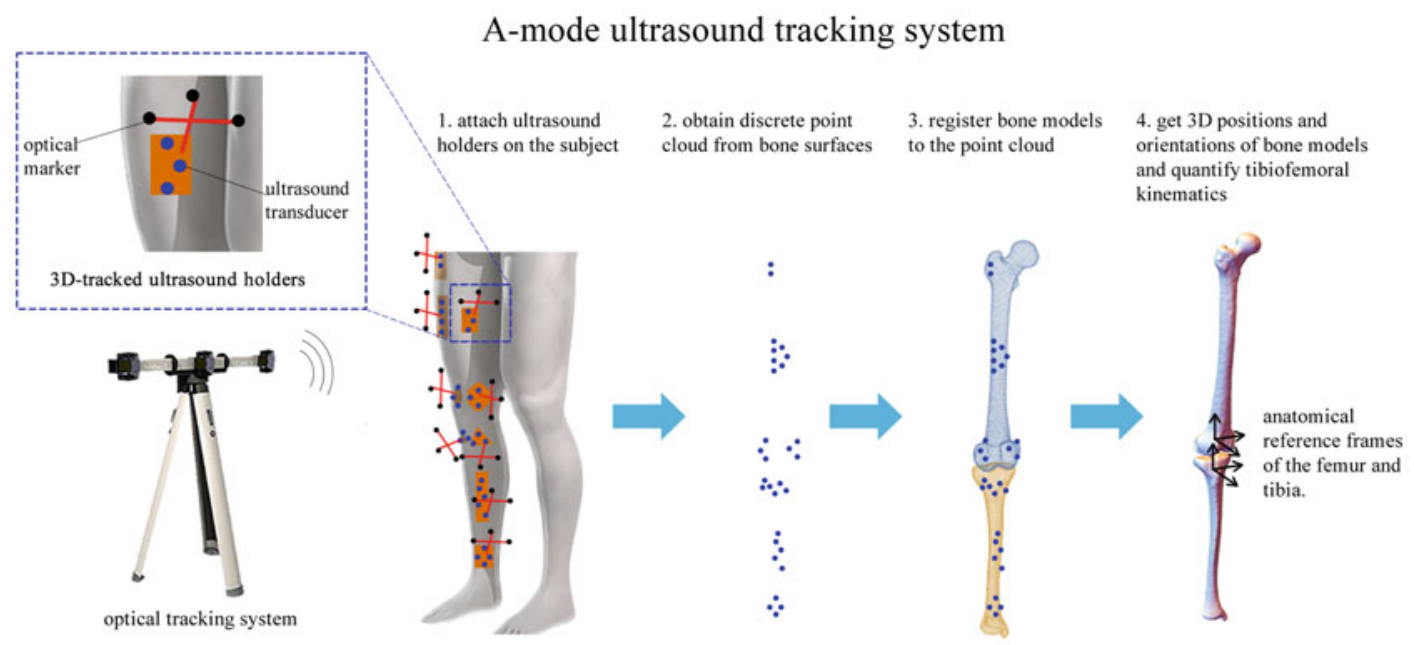

Fig. 11.3 A schematic representation of the A-mode ultrasound system to quantify tibiofemoral kinematics from obtained point cloud. Also shown is the placement of ultrasound holders on the right leg

focus at $2.5 \mathrm{~cm}$, Imasonic SAS, Voray/l'Ognon, France) and 27 active optical markers (tracked by Visualeyez system) were installed into the custom ultrasound holders. The ultrasound holders cover various anatomical areas on the lower extremity, including ankle, middle shaft of tibia, tibial condyles, femoral epicondyles, middle thigh, and great trochanter (Fig. 11.3). The ultrasound holders were designed in SolidWorks (Waltham, Massachusetts, USA) and manufactured using polyamide powder material in 3D printer (EOS Formiga P110, EOS GmbH, Krailling, Germany) to insure high accuracy on their $3 \mathrm{D}$ geometrical structures for maintaining the strength, rigidity, and stability. Therefore, the spatial relations between each A-mode ultrasound transducer and each optical marker were known parameters. Hence no further physical calibration is required as this is build-in design.

The US transducers attached to the customized ultrasound holders reproduce the 
necessary information (i.e., the 3D discrete point cloud) to reconstruct bone motion through the obtained raw ultrasound signals and spatial information. A brief description of this processing can be found in our previous paper [40]. The ultrasound detected point can be digitalized through the known origin and pointing direction of each ultrasound transducers and the related spatial relation between each optical marker, when the depth of bone surface is obtained. For each subject, the anatomical landmarks (ankle, middle shaft of tibia, tibial condyles, femoral epicondyles, middle thigh, great trochanter) were manually digitalized in the segmented bone models, which will be used in point cloud registration. The yielded discrete point cloud was fed to a registration algorithm, using a modified weighted iterative closest point algorithm [43, 44] to get the transformations from the original geometrical surface models to the actual 3D positions and orientations of bone models in the laboratory coordinate system. The raw ultrasound signals from 30 (15 for the femur and 15 for the tibia) A-mode ultrasound transducers and the raw 3D coordinates of 27 optical markers were synchronized and recorded at $45 \mathrm{~Hz}$ sample rate. Thus the $3 \mathrm{D}$ discrete point cloud was reproduced in $45 \mathrm{~Hz}$ sample rate during experiment. The respective tibiofemoral kinematics were derived from the method based on the ISB recommendations $[45,46]$.

\subsubsection{Experiments}

The ultrasound holders were attached to the right leg of each subject and were fixated by using skin tapes in order to cover all needed anatomical areas without any hindrance during movements. After attaching all ultrasound holders, each subject performed two sets of trials: (1) walking at three different imposed speeds $(1 \mathrm{~km} / \mathrm{h}, 2$ $\mathrm{km} / \mathrm{h}$, and $3 \mathrm{~km} / \mathrm{h}$ ) on the treadmill and (2) stair descent from two consecutive stairs (first stair, $18 \mathrm{~cm}$ height; second stair, $21 \mathrm{~cm}$ height, next to the ground). For treadmill walking, at least five gait cycles were recorded for each trial. For the stair-descent trial, each subject was asked to repeat three times for stair-descent trial and was always asked to step the right leg at first for each stair. It took about one and a half hours to complete an experiment of one subject, including attachment of ultrasound holders to the subject, the calibration procedure, and all measurements of all trials.

\subsubsection{Data Processing}

After all experiments, 3D knee joint motions and 6-DOF tibiofemoral kinematics were calculated for all trials over all gait cycles and three repeated stair-descent cycles. The calculated 6-DOF tibiofemoral kinematics of treadmill walking were averaged across five subjects under imposed three treadmill speeds. The mean and standard deviation across five subjects of calculated 6-DOF tibiofemoral kinematics for the stair-descent cycles were illustrated as the functions of percentage of two-stair descending (100\% represent one complete cycle of one-stair descending; thus completed cycle is $200 \%$ ).

To demonstrate the capability of detecting the bony surfaces from different anatomical areas and the capability of detecting the changes of depth of detected bone surface caused by soft tissue deformation, several M-mode (motion mode) ultrasound images were generated. Mmode image is defined as motion display of the ultrasound wave along a chosen ultrasound line (in our case, a single ultrasound transducer element) during a time period. Its $\mathrm{x}$-axis represents the number of samples. Its $y$-axis represents the intensity of received echo in a color map. It provides a two-dimensional view of the depth changes.

\subsection{Results}

The mean of 6-DOF tibiofemoral kinematics across five subjects under imposed three different treadmill speeds are illustrated in Fig. 11.4 The mean \pm standard deviation of 6-DOF tibiofemoral kinematics across five subjects during stair descending is shown in Fig. 11.5. 

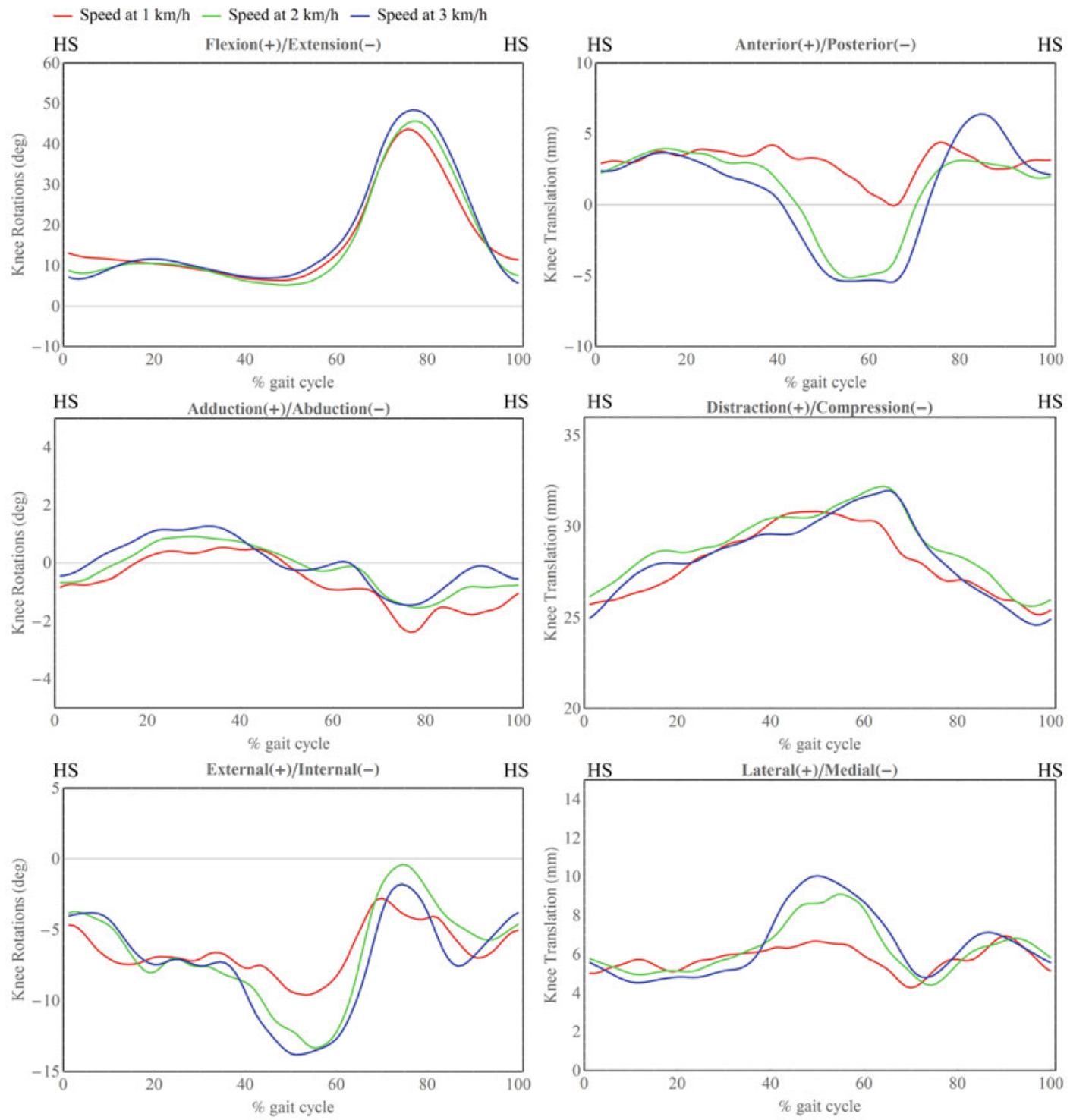

Fig. 11.4 Averaged 6-DOF tibiofemoral kinematics across five subjects for imposed three different speeds: $1 \mathrm{~km} / \mathrm{h}$ (red line), $2 \mathrm{~km} / \mathrm{h}$ (green line), and $3 \mathrm{~km} / \mathrm{h}$ (blue line)

\subsubsection{Treadmill Walking}

The largest rotation motion was flexionextension, followed by external-internal rotation and adduction-abduction. The peak knee flexion at the swing phase increased with increasing imposed speed. At heel strike, the knee was not fully extended (reach $0^{\circ}$ ) at all three imposed speeds. As the imposed treadmill speed increased, the extension angle of the knee joint increased at heel strike. The knee joint distraction started to increase from the heel strike and reached the peak until the swing phase started. Walking at the lowest imposed speed resulted in the smallest range of motion (ROM) for all 6DOF kinematics compared to a higher imposed speed. We illustrated the M-mode images in two groups: (1) several anatomical areas at thesame 

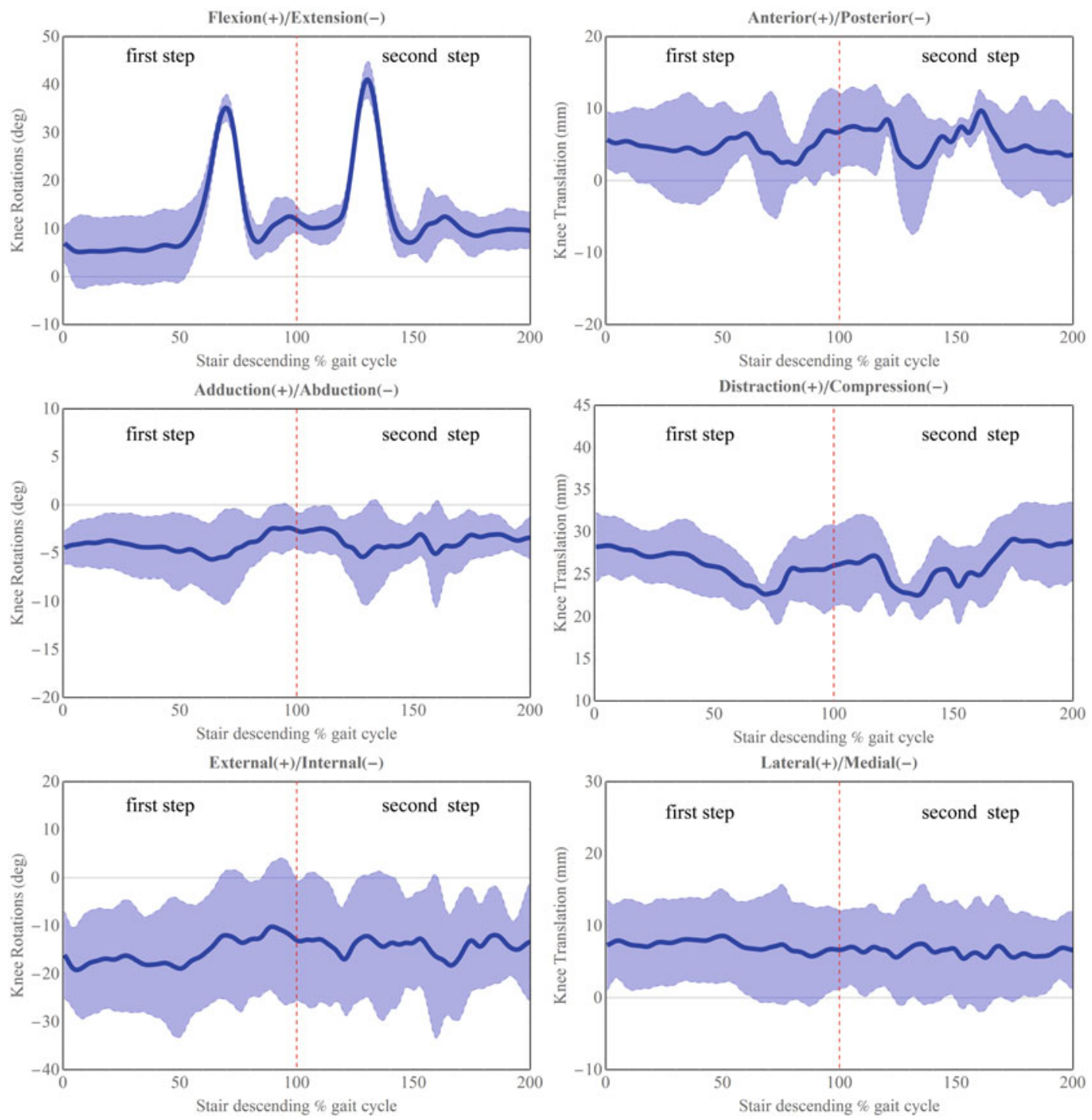

Fig. 11.5 6-DOF tibiofemoral kinematics for two consecutive stairs descending across five subjects. The solid line represents the mean data while the shaded areas represent \pm 1 standard deviation from the mean. One

hundred percent of stair descending cycle represents the completion of the first floor. Two hundred percent of stair descending cycle represents the completion of the second floor

treadmill speed (lateral side of middle femur, anterior side of middle femur, femoral lateral epicondyle, medial side of middle tibia at 1 $\mathrm{km} / \mathrm{h}$ ) and (2) three different treadmill speeds of an identical location (lateral side of middle femur at $1,2,3 \mathrm{~km} / \mathrm{h}$ ).

\subsubsection{Stair Descending}

The mean flexion angle across five subjects reached the first peak $\left(35.1^{\circ}\right)$ during stepping down the first stair $(18 \mathrm{~cm})$ and reached the second peak $\left(41.0^{\circ}\right)$ during stepping down the 


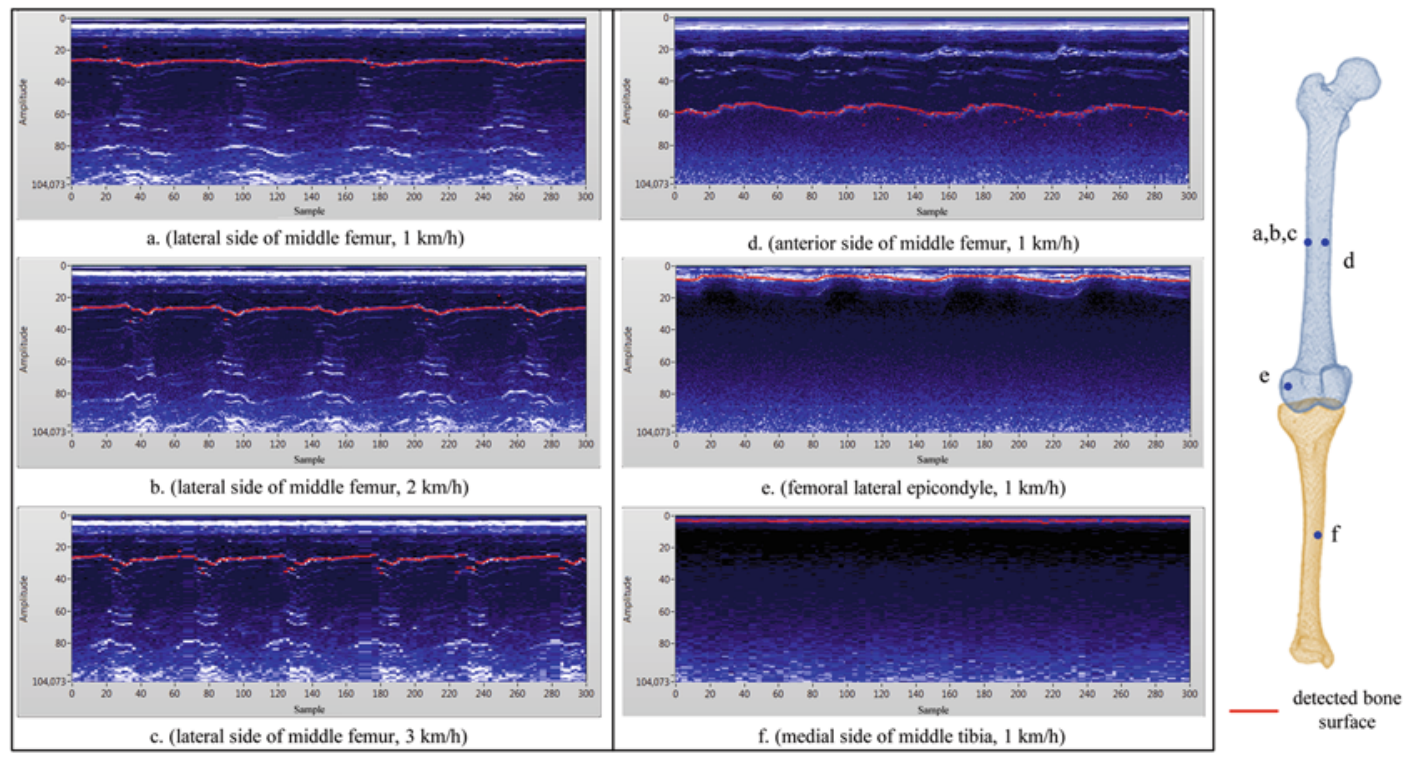

Fig. 11.6 Left: the examples of six M-mode images of one subject: $\mathrm{x}$-axis of M-mode image represents the number of samples, $\mathrm{y}$-axis of M-mode image represents the intensity of received ultrasound echo in a color map; $(\mathbf{a}, \mathbf{b}, \mathbf{c})$ the M-mode images for lateral side of middle femur location at $1 \mathrm{~km} / \mathrm{h}, 2 \mathrm{~km} / \mathrm{h}$, and $3 \mathrm{~km} / \mathrm{h}$, respectively;

second stair $(21 \mathrm{~cm})$. The knee joint distraction started to decrease when the right leg reached the next floor level and started to flex the knee to support the increasing pressure on the right knee. When the contralateral foot reached the same floor, the joint distraction began to increase until flexion angle was as the same as neutral standing. The similar changing pattern of joint distraction happened during stepping down to the second floor level.

\subsubsection{M-mode Images}

The examples of several M-mode images of one subject were illustrated in Fig. 11.6. The frequency of depth changing on the lateral side of middle femur was increased with the increase on the imposed treadmill speed. The changing range of depth also increased with the increases of speed. The depth changing at same speed was different for various anatomical locations. The depth changing of medial side of middle tibia (d) the M-mode image for anterior side of the middle femur at $1 \mathrm{~km} / \mathrm{h}$; (e) the M-mode image for femoral lateral epicondyle at $1 \mathrm{~km} / \mathrm{h}$; (e) the M-mode image for medial side of middle tibia at $1 \mathrm{~km} / \mathrm{h}$; right: the illustration of abovementioned anatomical locations on the femur and tibia

had the smallest, since the thickness of soft tissue is the smallest compared to other locations. The anterior and lateral sides of the middle femur had the large variation in detected bone depth, as the thickness of soft tissue is the largest to other location.

\subsection{Discussions}

We presented a novel method to dynamically track the knee joint motion and to quantify 6DOF tibiofemoral kinematics in a noninvasive and non-radiative manner. The combination of multiple A-mode ultrasound transducers with a conventional motion capture system provides an alternative method to capture skeletal motions and kinematics with mitigating the effect of STA. In this study, the in vivo capability of our proposed ultrasound tracking system to measure knee joint motion and to quantify 6-DOF tibiofemoral kinematics was demonstrated in two motor tasks of daily activities. The kinematic 
alterations caused by different gait parameters have also been identified by ultrasound tracking system. The peak flexion angle during swing phase on treadmill walking reduced apparently when participants walked at the slow imposed speed, which is in accordance with the findings in previous study [47]. Similarly, a smaller ROM was associated with a lower imposed speed during walking for all 6-DOF tibiofemoral kinematics [47]. The patterns of obtained 6-DOF tibiofemoral kinematics on the treadmill waking were in accordance with those of previous tibiofemoral kinematic outcome derived from a mobile fluoroscopy system [48]. For the stair descending, the peak flexion angle was correlated with the height of the stair level. The kinematic alterations caused by small changes of gait parameters could be recognized by our ultrasound tracking system, which proves a certain extent of sensitivity of ultrasound tracking system.

The novelty of this study lies in the secondary development of existed techniques, i.e., motion capture and ultrasound imaging. Taking advantage of ultrasound techniques extends the range of detection of a conventional motion capture system from superficial skin surface tracking to internal bony surface tracking. As a consequence, the sufficient spatial information (trajectories) of bony segments under the skin surface contributes to the accurate bone motion tracking and accurate kinematic estimation.

When comparing the ultrasound tracking system to the conventional skin-mounted markers measurement, the advantage is the removal of STA on the measurement data and its propagation on kinematic outcomes [49]. As demonstrated in the examples of M-mode images, A-mode ultrasound transducers has the capability of detecting the depth changing of bone surfaces on different anatomical areas. The capability could improve the validity of representing actual bone movement, since the trajectories of bone surfaces will be measured instead of superficial skin surfaces. A comparison with a skin-mounted marker measurement in a cadaveric setting has been conducted in our previous study. However, a critical comparison with a skin marker system under in vivo conditions is necessary, particularly if a ground truth method (e.g., an advanced mobile fluoroscopy system) $[28,29]$ can be incorporated. Currently, the FOV of our system is the same as the conventional motion capture systems, since it only depends on the FOV of the employed motion capture system. In addition, the length of the cables connected to the ultrasound transducers also restricts the maximum dynamic motion range. However, this aspect can be solved reasonably easy by extending the length of cables or employing an ambulant acquisition terminal instead of a stand-alone desktop computer on the side.

This work has several limitations:

Firstly, no "ground truth" measurement was employed during experiment. There is no a noninvasive and non-radiative method to obtain the ground truth of movements (walking and stair descent). Available methods like intracortical bone pins and fluoroscopic systems could potentially harm the subjects. An in vivo validation study will be completed in the near future so that the results would facilitate the improvements of current system and provide valuable comparisons with existed techniques. Secondly, only five healthy subjects were involved in this study. Ideally, a cohort of living subject covering different patients and healthy groups with different sizes and BMIs accompanied with a ground truth measurements as a reference (e.g., advanced mobile fluoroscopy system) [28, 29] could provide more valuable information with regard to the pathological patterns on kinematics.

Thirdly, a standardized definition of the femoral and tibial ARF across different subjects is imperative for 6-DOF joint kinematics analysis. Since the discrepancies of the defined femoral and tibial ARF among different subjects caused the deviations on all 6-DOF kinematic outcomes and patterns for various motor tasks. In further study, a standardized definition of femoral and tibia ARF across different subjects should be proposed in order to eliminate the intrinsic variations among defined femoral and tibial ARF. 
Fourthly, it has been shown that gait patterns on a treadmill are different to freely normal level walking [48]. However, in this study, the focus was on the demonstration of knee joint motion tracking during dynamic movements and detecting the kinematic alterations caused by different imposed treadmill speed and heights of staircase. Treadmill speed is a convenient parameter to change under a highly controlled scenario.

Fifthly, the cables and skin tapes may influence the nature gait pattern for individuals. In the future, we are aiming to develop a miniature and lightweight system toward a wearable measurement system that would facilitate its implementation in the clinic. Furthermore, future study will also focus on the improvement of designing the ultrasound holders in term of lighter, smaller, user friendly, and ergonomic design. These improvements on designs of ultrasound hold would be beneficial to popularize our system in a broader application field and to facilitate the usage among a cohort of subjects.

In summary, we developed an alternative, ultrasound tracking system that is capable of measuring knee joint motion. Hence, we conclude that this prototyping system has great potential to measure human kinematics in an ambulant, nonradiative, and noninvasive manner.

\section{References}

1. Ramsey DK, Wretenberg PF (1999) Biomechanics of the knee: methodological considerations in the in vivo kinematic analysis of the tibiofemoral and patellofemoral joint. Clin Biomech 14(9):595-611. https://doi.org/10.1016/S0268-0033(99)00015-7

2. Schilling C, Krüger S, Grupp TM, Duda GN, Blömer W, Rohlmann A (2011) The effect of design parameters of dynamic pedicle screw systems on kinematics and load bearing: an in vitro study. Eur Spine J 20(2):297-307. https://doi.org/10.1007/s00586-010-1620-6

3. Simon D. What is "registration" and why is it so important in CAOS

4. Sugano N, Sasama T, Sato Y, Nakajima Y, Nishii $\mathrm{T}$, Yonenobu K et al (2001) Accuracy evaluation of surface-based registration methods in a computer navigation system for hip surgery performed through a posterolateral approach. Comput Aided Surg 6(4):195-203. https://doi.org/10.1002/igs.10011

5. Anderson KC, Buehler KC, Markel DC (2005) Computer assisted navigation in total knee arthroplasty: comparison with conventional methods. J Arthroplasty 20(7 Suppl 3):132-138. https://doi.org/10.1016/j.arth.2005.05.009

6. Mavrogenis AF, Savvidou OD, Mimidis G, Papanastasiou J, Koulalis D, Demertzis N et al (2013) Computer-assisted navigation in orthopedic surgery. Orthopedics 36(8):631-642. https://doi.org/10.3928/01477447-20130724-10

7. Kaiser JM, Vignos MF, Kijowski R, Baer G, Thelen DG (2017) Effect of Loading on In Vivo Tibiofemoral and Patellofemoral Kinematics of Healthy and ACL-Reconstructed Knees. Am J Sports Med 45(14):3272. https://doi.org/10.1177/0363546517724417

8. Zeng X, Ma L, Lin Z, Huang W, Huang Z, Zhang $Y$ et al (2017) Relationship between KellgrenLawrence score and 3D kinematic gait analysis of patients with medial knee osteoarthritis using a new gait system. Sci Rep 7(1):4080. https://doi.org/10.1038/s41598-017-04390-5

9. Delp SL, Anderson FC, Arnold AS, Loan P, Habib A, John CT et al (2007) OpenSim: open-source software to create and analyze dynamic simulations of movement. IEEE Trans Biomed Eng 54(11):19401950. https://doi.org/10.1109/tbme.2007.901024

10. Gerus P, Sartori M, Besier TF, Fregly BJ, Delp SL, Banks SA et al (2013) Subjectspecific knee joint geometry improves predictions of medial tibiofemoral contact forces. J Biomech 46(16):2778-2786. https://doi.org/10.1016/j.jbiomech.2013.09.005

11. Fuller J, Liu LJ, Murphy MC, Mann RW (1997) A comparison of lower-extremity skeletal kinematics measured using skin- and pinmounted markers. Hum Mov Sci 16(2-3):219-242. https://doi.org/10.1016/S0167-9457(96)00053-X

12. Richard V, Cappozzo A, Dumas R (2017) Comparative assessment of knee joint models used in multi-body kinematics optimisation for soft tissue artefact compensation. J Biomech. https://doi.org/10.1016/j.jbiomech.2017.01.030

13. Andersen MS, Benoit DL, Damsgaard M, Ramsey DK, Rasmussen J (2010) Do kinematic models reduce the effects of soft tissue artefacts in skin marker-based motion analysis? An in vivo study of knee kinematics. J Biomech 43(2):268-273. https://doi.org/10.1016/j.jbiomech.2009.08.034

14. Lafortune MA, Cavanagh PR, Sommer HJ, Kalenak A (1992) Three-dimensional kinematics of the human knee during walking. J Biomech 25(4):347-357. https://doi.org/10.1016/0021-9290(92)90254-X

15. Cereatti A, Bonci T, Akbarshahi M, Aminian K, Barre A, Begon M et al (2017) Standardization proposal of soft tissue artefact description for data sharing in human motion measurements. J Biomech. https://doi.org/10.1016/j.jbiomech.2017.02.004 
16. Akbarshahi $M$, Schache AG, Fernandez JW, Baker R, Banks S, Pandy MG (2010) Noninvasive assessment of soft-tissue artifact and its effect on knee joint kinematics during functional activity. J Biomech 43(7):1292-1301. https://doi.org/10.1016/j.jbiomech.2010.01.002

17. Benoit DL, Ramsey DK, Lamontagne M, $\mathrm{Xu}$ L, Wretenberg P, Renström P (2006) Effect of skin movement artifact on knee kinematics during gait and cutting motions measured in vivo. Gait Posture 24(2):152-164. https://doi.org/10.1016/j.gaitpost.2005.04.012

18. Bonnet V, Richard V, Camomilla V, Venture G, Cappozzo A, Dumas R (2017) Joint kinematics estimation using a multi-body kinematics optimisation and an extended Kalman filter, and embedding a soft tissue artefact model. J Biomech. https://doi.org/10.1016/j.jbiomech.2017.04.033

19. Cappozzo A, Cappello A, Croce UD, Pensalfini F (1997) Surface-marker cluster design criteria for 3-D bone movement reconstruction. IEEE Trans Biomed Eng 44(12):1165-1174. https://doi.org/10.1109/10.649988

20. Andersen MS, Damsgaard M, Rasmussen J (2009) Kinematic analysis of over-determinate biomechanical systems. Comput Methods Biomech Biomed Eng 12(4):371-384. https://doi.org/10.1080/10255840802459412

21. Bonnechère $B$, Sholukha $V$, Salvia $P$, Rooze M, Van Sint Jan S (2015) Physiologically corrected coupled motion during gait analysis using a model-based approach. Gait Posture 41(1):319-322. https://doi.org/10.1016/j.gaitpost.2014.09.012

22. Charlton IW, Tate P, Smyth P, Roren L (2004) Repeatability of an optimised lower body model. Gait Posture 20(2):213-221. https://doi.org/10.1016/j.gaitpost.2003.09.004

23. Duprey S, Cheze L, Dumas R (2010) Influence of joint constraints on lower limb kinematics estimation from skin markers using global optimization. J Biomech 43(14):2858-2862. https://doi.org/10.1016/j.jbiomech.2010.06.010

24. Lu TW, O'Connor JJ et al (1999) J Biomech 32(2):129-134. https://doi.org/10.1016/S0021-9290(98)00158-4

25. Bingham J, Li G (2006) An optimized image matching method for determining in-vivo TKA kinematics with a dual-orthogonal fluoroscopic imaging system. J Biomech Eng 128(4):588-595. https://doi.org/10.1115/1.2205865

26. Baka N, Kaptein BL, Giphart JE, Staring M, de Bruijne M, Lelieveldt BPF et al (2014) Evaluation of automated statistical shape model based knee kinematics from biplane fluoroscopy. J Biomech 47(1):122-129. https://doi.org/10.1016/j.jbiomech.2013.09.022

27. Gray HA, Guan S, Pandy MG (2017) Accuracy of mobile biplane X-ray imaging in measuring 6-degree-of-freedom patellofemoral kinematics during overground gait. J Biomech 57:152-156. https://doi.org/10.1016/j.jbiomech.2017.04.009
28. Guan S, Gray HA, Keynejad F, Pandy MG (2016) Mobile biplane $\mathrm{x}$-ray imaging system for measuring 3D dynamic joint motion during overground gait. IEEE Trans Med Imaging 35(1):326-336. https://doi.org/10.1109/TMI.2015.2473168

29. List R, Postolka B, Schutz P, Hitz M, Schwilch P, Gerber H et al (2017) A moving fluoroscope to capture tibiofemoral kinematics during complete cycles of free level and downhill walking as well as stair descent. PLoS One 12(10):e0185952. https://doi.org/10.1371/journal.pone.0185952

30. Mazzoli V, Schoormans J, Froeling M, Sprengers AM, Coolen BF, Verdonschot N et al (2017) Accelerated 4D self-gated MRI of tibiofemoral kinematics. NMR Biomed. https://doi.org/10.1002/nbm.3791

31. Clarke EC, Martin JH, d'Entremont AG, Pandy MG, Wilson DR, Herbert RD (2015) A non-invasive, 3D, dynamic MRI method for measuring muscle moment arms in vivo: Demonstration in the human ankle joint and Achilles tendon. Med Eng Phys 37(1):93-99. https://doi.org/10.1016/j.medengphy.2014.11.003

32. Kaiser J, Bradford R, Johnson K, Wieben O, Thelen DG (2013) Measurement of 3D tibiofemoral kinematics using volumetric SPGR-VIPR Imaging. Magn Reson Med 69(5):1310-1316. https://doi.org/10.1002/mrm.24362

33. Forsberg D, Lindblom M, Quick P, Gauffin H (2016) Quantitative analysis of the patellofemoral motion pattern using semi-automatic processing of 4D CT data. Int J Comput Assist Radiol Surg 11(9):17311741. https://doi.org/10.1007/s11548-016-1357-8

34. Zhao K, Breighner R, Holmes D, Leng S, McCollough C, An K-N (2015) A technique for quantifying wrist motion using four-dimensional computed tomography: approach and validation. J Biomech Eng 137(7):0745011-0745015. https://doi.org/10.1115/1.4030405

35. Smistad E, Falch TL, Bozorgi M, Elster AC, Lindseth F (2015) Medical image segmentation on GPUs - A comprehensive review. Med Image Anal 20(1):1-18. https://doi.org/10.1016/j.media.2014.10.012

36. Wein W, Karamalis A, Baumgartner A, Navab N (2015) Automatic bone detection and soft tissue aware ultrasound-CT registration for computer-aided orthopedic surgery. Int J Comput Assist Radiol Surg 10(6):971-979. https://doi.org/10.1007/s11548-015-1208-Z

37. Fieten L, Schmieder K, Engelhardt M, Pasalic L, Radermacher K, Heger S (2009) Fast and accurate registration of cranial $\mathrm{CT}$ images with A-mode ultrasound. Int J Comput Assist Radiol Surg 4(3):225237. https://doi.org/10.1007/s11548-009-0288-z

38. Talib H, Peterhans M, Garcia J, Styner M, Gonzalez Ballester MA (2011) Information filtering for ultrasound-based real-time registration. IEEE Trans Biomed Eng 58(3):531-540. https://doi.org/10.1109/TBME.2010.2063703

39. Otake Y, Armand M, Armiger RS, Kutzer MD, Basafa E, Kazanzides P et al (2012) Intraoperative image-based multiview 2D/3D registration 
for image-guided orthopaedic surgery: incorporation of fiducial-based C-arm tracking and GPUacceleration. IEEE Trans Med Imaging 31(4):948962. https://doi.org/10.1109/TMI.2011.2176555

40. Niu K, Sluiter V, Sprengers A, Homminga J, Verdonschot N (eds) (2017) A novel tibiafemoral kinematics measurement system based on multi-channel a-mode ultrasound system. In: CAOS 2017. 17th annual meeting of the international society for computer assisted orthopaedic surgery; 2017 June 13, EasyChair, Aachen

41. Miranda DL, Rainbow MJ, Leventhal EL, Crisco JJ, Fleming BC (2010) Automatic determination of anatomical coordinate systems for threedimensional bone models of the isolated human knee. J Biomech 43(8):1623-1626. https://doi.org/10.1016/j.jbiomech.2010.01.036

42. Inc. PPT. VZ4000v technical specifications. http:// www.ptiphoenix.com/products/trackers/VZ4000v. Accessed 3 Mar 2017

43. Maurer CR Jr, Maciunas RJ, Fitzpatrick JM (1998) Registration of head CT images to physical space using a weighted combination of points and surfaces. IEEE Trans Med Imaging 17(5):753-761. https://doi.org/10.1109/42.736031

44. Besl PJ, McKay HD (1992) A method for registration of 3-D shapes. IEEE Trans Pattern Anal Mach Intell 14(2):239-256. https://doi.org/10.1109/34.121791
45. Wu G, Cavanagh PR (1995) ISB recommendations for standardization in the reporting of kinematic data. J Biomech 28(10):1257-1261. https://doi.org/10.1016/0021-9290(95)00017-C

46. Grood ES, Suntay WJ (1983) A joint coordinate system for the clinical description of three-dimensional motions: application to the knee. J Biomech Eng 105(2):136-144

47. Mannering $\mathrm{N}$, Young $\mathrm{T}$, Spelman $\mathrm{T}$, Choong PF (2017) Three-dimensional knee kinematic analysis during treadmill gait: Slow imposed speed versus normal selfselected speed. Bone Joint Res 6(8):514-521. https://doi.org/10.1302/2046-3758.68.bjr-2016-0296.r1

48. Guan S, Gray HA, Schache AG, Feller J, de Steiger R, Pandy MG (2017) In vivo sixdegree-of-freedom knee-joint kinematics in overground and treadmill walking following total knee arthroplasty. J Orthop Res 35:1634-1643. https://doi.org/10.1002/jor.23466

49. Jia R, Monk P, Murray D, Noble JA, Mellon S (2017) CAT \& MAUS: A novel system for true dynamic motion measurement of underlying bony structures with compensation for soft tissue movement. J Biomech. https://doi.org/10.1016/j.jbiomech.2017.04.015 